\title{
Actitudes Hacia los Adultos Mayores en Universitarios Mexicanos: Comparaciones por Género
}

\author{
Dr. Carolina Jiménez Lira, \\ Dr. Ma. Concepción Soto, \\ Dr. Yunuen Rangel, \\ Fernando Mondaca, PhD \\ Miguel Conchas, M.C. \\ Dr. Susana Ivonne Aguirre, \\ Dr. Raúl Josué Nájera,
}

Facultad de Ciencias de la Cultura Física, Universidad Autónoma de Chihuahua, México

Doi:10.19044/esj.2019.v15n32p160 URL:http://dx.doi.org/10.19044/esj.2019.v15n32p160

\section{Resumen}

El objetivo de esta investigación consistió en comparar los perfiles de actitud hacia los adultos mayores de alumnos y alumnas universitarios mexicanos. La muestra total fue de 1634 sujetos; 841 mujeres y 793 hombres, con una edad media de 20.48 años $(\mathrm{DE}=1.73)$ y 20.86 años $(\mathrm{DE}=1.88)$ respectivamente. El abordaje adoptado en la investigación se enmarcó dentro de un enfoque cuantitativo con un diseño descriptivo tipo encuesta. Los resultados del análisis multivariante de la varianza, seguido por los análisis de varianza univariados, revelan que las mujeres son quienes muestran una actitud más positiva hacia los adultos mayores en los factores esfera personal y esfera social, mientras que en lo que se refiere al factor esfera emocional no se encontraron diferencias. Las diferencias encontradas entre hombres y mujeres con respecto a su actitud hacia el adulto mayor, sugieren que al diseñar cualquier tipo de intervención que tenga como objetivo mejorar dicha actitud habrá que tomar en cuenta la variable género. Futuras investigaciones deberían replicar estos hallazgos en muestras más amplias.

Palabras clave: Actitudes, Género, Autopercepciones 


\title{
Attitudes Towards Older Adults in Mexican University Students: Comparisons by Gender
}

\author{
Dr. Carolina Jimenez Lira, \\ Dr. Ma. Concepcion Soto, \\ Dr. Yunuen Rangel, \\ Fernando Mondaca, PhD \\ Miguel Conchas, M.C. \\ Dr. Susana Ivonne Aguirre, \\ Dr. Raul Josue Najera,
}

Facultad de Ciencias de la Cultura Física, Universidad Autónoma de Chihuahua, México

\begin{abstract}
This paper focuses on comparing the attitude profiles towards older adults of Mexican university students. The total sample of the study was 1634 subjects, 841 women and 793 men, with an average age of 20.48 years (SD = $1.73)$ and 20.86 years $(S D=1.88)$ respectively. The approach adopted in the research was framed within a quantitative approach with a descriptive design type survey. The results of the multivariate analysis of variance, followed by the univariate analysis of variance, revealed that women are the ones who show a more positive attitude towards older adults in the factors personal sphere and social sphere. On the other hand, in what refers to the emotional sphere factor, no differences were found. The differences found between men and women, with respect to their attitude towards the elderly, suggest that when designing any type of intervention that aims to improve this attitude, the gender variable has to be taken into account. Future researches should replicate these findings on a wider sampling.
\end{abstract}

Keywords: Attitudes, Gender, Self-perceptions

\section{Introducción}

La etapa de la vejez está asociada con actitudes negativas como fragilidad, rigidez y dependencia, y con atributos positivos tales como sabiduría, longevidad y aumento de la experiencia o logros personales. Tales actitudes frente al envejecimiento están vinculadas con los estereotipos de edad, dando lugar a los prejuicios y a la discriminación por edad y 
comportamientos ageístas. Tales estereotipos operan inconscientemente y se interiorizan a lo largo de la vida; a menudo, las personas mayores incorporan estos estereotipos en su propio pensamiento y autopercepción, los cuales intervienen de manera significativa en el bienestar cognitivo y funcional, exacerbando la enfermedad y la discapacidad alcanzando una mayor influencia negativa en los comportamientos (Bennett \& Gaines, 2010; Levy, 2003, 2009; Levy, Ferrucci, Zonderman, Slade, Troncoso \& Resnick, 2016).

Desde esta perspectiva, autores como Diehl et al. (2014), Dionigi (2015), Kotter-Grühn, Kornadt y Stephan (2016), señalan que las actitudes hacia el envejecimiento se consideran elementos críticos para el ajuste y la supervivencia de las personas mayores, debido a que contribuyen a su adaptación y refuerzan la forma en cómo los individuos más jóvenes perciben y experimentan su propio proceso de envejecimiento. Levy (2003) en su revisión, muestra como en los individuos más jóvenes los estereotipos sobre el envejecimiento se interiorizan, para luego, cuando los individuos alcanzan la vejez, convertirse en auto estereotipos. Del mismo modo, Rupp, Vodanovich y Credé (2005) observaron que hombres jóvenes obtuvieron puntuaciones significativamente más altas de ageísmo que las personas mayores y las mujeres.

En esta misma línea, Kornadt, Voss y Rothermund (2015) descubrieron que los efectos de interiorización de los estereotipos de edad fueron más fuertes en los grupos de jóvenes de mediana edad y emergieron en los dominios de la familia, la personalidad, el trabajo y el ocio, mientras que, Levy, Zonderman, Slade y Ferrucci (2009) afirman que los estereotipos negativos de edad en individuos de 18 a 49 años aumentan la predicción de múltiples enfermedades cardiovasculares. Finalmente Serrani (2011) documentó que en individuos jóvenes los estereotipos negativos llegan a representar un obstáculo para el desarrollo de conocimientos y la empatía requeridas para asistir a las personas mayores (ver también Blanco-Molina \& Pinazo-Hernandiz, 2016).

Tales actitudes discriminatorias impactan negativamente en la autopercepción, la salud y bienestar de las personas (Franco, Villarreal, Vargas, Martínez \& Galicia, 2010; Kotter-Grühn \& Hess, 2012; Kotter-Grühn et al., 2016); en los adultos mayores estos puntos de vista negativos impactan en las creencias de autoeficacia y autocontrol sobre su rendimiento en la memoria (Hughes, Geraci y De Forrest, 2013) y funcionamiento cognitivo (Robertson, King-Kallimanis \& Kenny, 2016).

Rothermund y Brandtstädter (2003) encontraron en un estudio longitudinal que las personas mayores con las creencias negativas sobre la vejez y el envejecimiento mostraron un deterioro de su autoconcepto en un intervalo de ocho años, mientras que las opiniones positivas sobre el envejecimiento predijeron una mejor autoevaluación. 
Autores como Coudin y Alexopoulos (2010), Kotter-Grühn, Neupert y Stephan, (2015), sugieren que la activación de estereotipos negativos en los adultos mayores puede tener efectos nocivos en la autoevaluación y funcionamiento, asociándose con deterioro físico y mental, y a su vez pueden contribuir a conductas de dependencia y fomentar mayores sentimientos de soledad, bajos niveles de extraversión y limitaciones para asumir riesgos, mientras que las actitudes positivas hacia el envejecimiento en la vida adulta se relacionan con indicadores de un mejor funcionamiento físico, mejor salud, mayor calidad de vida y mayor longevidad (Sargent-Cox, Anstey \& Luszcz, 2012; Stephan, Chalabaev, Kotter-Grühn \& Jaconelli, 2012; Stephan, Sutin \& Terracciano, 2015).

Por otra parte, las malas expectativas y percepciones del envejecimiento están asociados igualmente con una menor probabilidad de desarrollar comportamientos de salud preventivos en los adultos mayores (Sarkisian, Prohaska, Wong, Hirsch \& Mangione, 2005); por el contrario, tener una actitud positiva al envejecimiento contribuye a mejoras en la autopercepción sobre la salud física y mental, asociándose con mayores niveles de satisfacción con la vida (Bryant et al., 2012; Castellano, 2014), niveles más bajos de ansiedad y depresión (Bryant et al., 2014), y estudios reportados por Levy, Slade, Murphy y Gill (2012) señalan que los adultos mayores con estereotipos positivos fueron más propensos a recuperarse por completo de la discapacidad severa que aquellos con los estereotipos negativos de edad.

La superación de los estereotipos negativos del envejecimiento a través de cambios a nivel social e individual puede ayudar a promover un envejecimiento exitoso (Bryant et al., 2012). Los hallazgos sugieren que actitudes positivas puede tener un efecto protector de la disminución en el funcionamiento físico en la vejez y tener una actitud positiva hacia el envejecimiento contribuye a resultados físicos y mentales saludables (Freeman, Santini, Tyrovolas, Rummel-Kluge \& Koyanagi, 2016; SargentCox, Anstey \& Luszcz, 2013).

En este contexto, nuestro interés es estudiar las actitudes hacia los adultos mayores por parte de hombres y mujeres universitarios mexicanos mediante la escala de actitudes hacia el adulto mayor de Kogan (1961) versión en español de Aguirre, Ornelas, Benavides, Gastélum y Mondaca (en prensa), la cual presenta características que favorecen su elección como su facilidad de administración y economía de tiempo, su extenso uso como referencia para la construcción y validación de otros instrumentos, así como sus buenas propiedades psicométricas en relación con la validez de contenido, validez de constructo, consistencia interna y fiabilidad test-retest, mostrando estabilidad estructural de sus dimensiones. 
En este estudio se intenta determinar las diferencias y similitudes entre hombres y mujeres universitarios mexicanos en cuanto a su actitud hacia los adultos mayores, tomando en cuenta su importancia para el bienestar psicológico personal. En consecuencia, esta investigación pretende, como investigación aplicada, aportar información que se traduzca en una práctica educativa de mayor calidad en el contexto de atención a la diversidad; contribuyendo al saber pedagógico en el esclarecimiento de los factores que conforman un modelo de desarrollo humano integral.

\section{- Método}

\section{Participantes}

Participaron en el estudio 1634 sujetos, 841 mujeres y 793 hombres estudiantes universitarios de la ciudad de Chihuahua México. La edad de los sujetos fluctuó entre los 18 y 25 años, con $M=20.48 \pm 1.73$ años para las mujeres y $M=20.86 \pm 1.88$ años para los hombres.

La muestra se obtuvo mediante un muestreo por conveniencia, tratando de abarcar la representatividad de las diferentes licenciaturas que se ofrecen en la Facultad de Ciencias de la Cultura Física de la Universidad Autónoma de Chihuahua.

\section{Instrumento}

Se utilizó la escala de actitudes hacia el adulto mayor de Kogan (KAOP) versión en español de Aguirre et al. (en prensa). También se empleó un cuestionario tipo Likert, asistido por computadora, que consta de 12 ítems agrupados en tres dimensiones o subescalas (esfera personal, esfera social y esfera emocional). En este cuestionario el encuestado manifiesta su grado de acuerdo con cada uno de los ítems propuestos, eligiendo entre once posibles respuestas: completamente en desacuerdo (0), en desacuerdo $(1,2$, y 3), ni de acuerdo ni en desacuerdo (4, 5 y 6$)$, de acuerdo ( 7,8 y 9) y completamente de acuerdo (10) (Figura 1). 


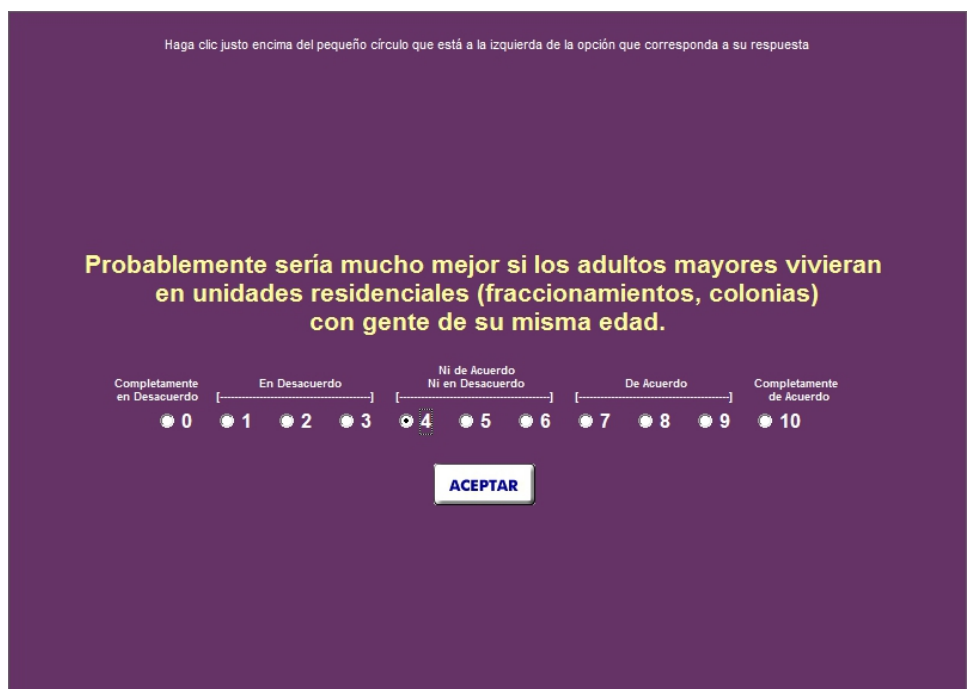

Figura 1. Ejemplo de respuesta para los ítems del cuestionario.

\section{Diseño}

En cuanto al diseño del estudio, se utilizó un enfoque cuantitativo con un diseño descriptivo y transversal tipo encuesta (Hernández, Fernández \& Baptista, 2010). La variable independiente fue el género y las variables dependientes el promedio de las puntuaciones en los factores esfera personal, esfera social y esfera emocional de la versión en español de Aguirre et al. (en prensa) para la escala de actitudes hacia el adulto mayor de Kogan (KAOP).

\section{Procedimiento}

Se invitó a participar en el estudio a los alumnos de las licenciaturas que se ofrecen en la Facultad de Ciencias de la Cultura Física (FCCF) de la Universidad Autónoma de Chihuahua. Los que aceptaron participar firmaron la carta de aceptación correspondiente. Luego se aplicó el instrumento antes descrito por medio de una computadora personal (módulo administrador del instrumento del editor de escalas de ejecución típica), en una sesión de aproximadamente 20 minutos en los laboratorios o centros de cómputo de la FCCF.

Al inicio de cada sesión se hizo una introducción sobre la importancia de la investigación y de cómo acceder al instrumento. Se les solicitó la máxima sinceridad y se les garantizó la confidencialidad de los datos que se obtuvieran. Las instrucciones de cómo responder se encontraban en las primeras pantallas, antes del primer reactivo del instrumento. Al término de la sesión se les agradeció su participación. Una vez aplicado el instrumento se procedió a recopilar los resultados por medio del módulo generador de resultados del editor de escalas versión 2.0 (Blanco et al., 2013). 


\section{Análisis de datos}

Se realizaron análisis estadísticos descriptivos (medias y desviaciones estándar) para todas las variables dependientes. Posteriormente, después de verificar que los datos cumplían con los supuestos de los análisis estadísticos paramétricos, se llevó a cabo un análisis multivariante de varianza (MANOVA), seguido por los análisis de varianza univariados (ANOVA), para examinar las diferencias entre mujeres y hombres universitarios en cuanto a sus actitudes hacia el adulto mayo. El tamaño del efecto se estimó mediante el eta cuadrado $\left(\eta^{2}\right)$. Todos los análisis estadísticos se realizaron con el programa SPSS versión 21.0 para Windows. El nivel de significación estadística se estableció en $p=.05$.

\section{- Resultados}

La Tabla 1 muestra los valores de las medias y las desviaciones estándar de las puntuaciones en los factores de actitudes hacia el adulto mayor, así como los resultados del MANOVA y los subsiguientes ANOVA. Los resultados del MANOVA mostraron diferencias globales estadísticamente significativas de acuerdo a la variable género en los puntajes promedio de los factores de actitudes hacia el adulto mayor (Wilks' $\lambda=.942 ; p<.001 ; \eta^{2}=$ .058). Posteriormente, los resultados de los ANOVA mostraron que las mujeres, en comparación con los hombres, reportan mejor actitud hacia el adulto mayor en los factores esfera personal $(F=88.066, p<.001)$ y esfera social $(F=15.230, p<.001)$ y no se observaron diferencias significativas en cuanto al factor esfera emocional.

Tabla 1. Resultados MANOVA y ANOVA para los puntajes promedio de los factores de actitud hacia el adulto mayor de acuerdo al género.

\begin{tabular}{|llllll|}
\hline \multicolumn{1}{c}{$\begin{array}{c}\text { Variables } \\
\text { Dependientes }\end{array}$} & $\begin{array}{l}\text { Mujeres } \\
(n=841)\end{array}$ & $\begin{array}{l}\text { Hombres } \\
(n=793)\end{array}$ & $\mathrm{F}$ & $\mathrm{p}$ & $\eta^{2}$ \\
\hline Esfera personal & $6.79(1.81)$ & $5.93(1.87)$ & 88.066 & $<.001$ & .051 \\
Esfera social & $4.97(1.86)$ & $4.59(2.01)$ & 15.230 & $<.001$ & .009 \\
Esfera emocional & $3.65(1.77)$ & $3.77(1.65)$ & 2.020 & $>.05$ & .001 \\
\hline
\end{tabular}

Nota. Los valores descriptivos se presentan como media (desviación estándar)

\section{- Conclusiones}

A partir de los resultados mostrados y de su análisis, se pueden obtener las siguientes conclusiones:

1. Los resultados revelan que en general las mujeres, en comparación con los hombres, muestran un perfil de actitud más positivo hacia los adultos mayores. Específicamente en lo relacionado con la apariencia 
personal de los adultos mayores (esfera personal), así como en lo relacionado con la convivencia con ellos (esfera social).

2. Las diferencias encontradas entre mujeres y hombres universitarios con respecto a su actitud hacia los adultos mayores sugieren además que al diseñar cualquier tipo de intervención que tenga como potencial cualquier mejora en dicha actitud habrá que tomar en cuenta a la variable género; no obstante, es preciso desarrollar más investigación al respecto pues el tema trasciende del todo los alcances de la presente investigación.

3. Se subraya además la importancia de realizar un mayor número de investigaciones sobre el tema en nuestro país.

Por último, cuando menos dos limitaciones están presentes en este trabajo. La primera es que los participantes son solo estudiantes, lo que supone una amenaza para la posibilidad de generalizar estos resultados. Ampliar la muestra (agregando por ejemplo jóvenes que no estudian) es un área de trabajo de cara al futuro. La segunda limitación proviene del propio instrumento de medición, que se basa en el autoinforme y que por ello puede contener los sesgos que se derivan de la deseabilidad social.

\section{References:}

1. Aguirre, S., Ornelas, M., Benavides, E. V., Gastélum, G. \& Mondaca, F. (en prensa). Composición factorial de la Escala de actitudes hacia el adulto mayor de Kogan en universitarios mexicanos. Revista Interamericana de psicologia, 53, 1-13.

2. Bennett, T. \& Gaines, J. (2010). Believing what you hear: The impact of aging stereotypes upon the old. Educational Gerontology, 36(5), 435-445.

3. Blanco-Molina, M. \& Pinazo-Hernandis, S. (2016). Información y exposición indirecta para reducir estereotipos hacia el envejecimiento. International Journal of Developmental and Educational Psychology. Revista INFAD de Psicología., 1(2), 367-380.

4. Blanco, H., Ornelas, M., Tristán, J. L., Cocca, A., Mayorga-Vega, D., López-Walle, J. \& Viciana, J. (2013). Editor for creating and applying computerise surveys. Procedia Social and Behavioral Sciences, 106, 935-940. doi: http://dx.doi.org/10.1016/j.sbspro.2013.12.105

5. Bryant, C., Bei, B., Gilson, K., Komiti, A., Jackson, H. \& Judd, F. (2012). The relationship between attitudes to aging and physical and mental health in older adults. International Psychogeriatrics, 24(10), 1674-1683. 
6. Bryant, C., Bei, B., Gilson, K., Komiti, A., Jackson, H. \& Judd, F. (2014). Antecedents of attitudes to aging: a study of the roles of personality and well-being. The Gerontologist, 00(00), 1-11.

7. Castellano, C. (2014). La influencia del apoyo social en el estado emocional y las actitudes hacia la vejez y el envejecimiento en una muestra de ancianos. International Journal of Psychology and Psychological Therapy, 14(3), 365-377.

8. Coudin, G. \& Alexopoulos, T. (2010). 'Help me! I'm old!'How negative aging stereotypes create dependency among older adults. Aging \& Mental Health, 14(5), 516-523.

9. Diehl, M., Wahl, H.-W., Barrett, A., Brothers, A., Micheb, M., Montepare, J., . . . Wurmf, S. (2014). Awareness of aging: Theoretical considerations on an emerging concept. Developmental Review, 34(2), 93-113.

10. Dionigi, R. A. (2015). Stereotypes of aging: Their effects on the health of older adults. Journal of Geriatrics, 2015.

11. Franco, M., Villarreal, E., Vargas, E., Martínez, L. \& Galicia, L. (2010). Estereotipos negativos de la vejez en personal de salud de un hospital de la Ciudad de Querétaro, México. Revista médica de Chile, 138(8), 988-993.

12. Freeman, A. T., Santini, Z. I., Tyrovolas, S., Rummel-Kluge, C., Haro, J. M., \& Koyanagi, A. (2016). Negative perceptions of ageing predict the onset and persistence of depression and anxiety: Findings from a prospective analysis of the Irish Longitudinal Study on Ageing (TILDA). Journal of Affective Disorders, 199, 132-138.

13. Hernández, R., Fernández, C. \& Baptista, P. (2010). Metodología de la investigación. México: McGraw- Hill.

14. Hughes, M., Geraci, L. \& De Forrest, R. (2013). Aging 5 years in 5 minutes the effect of taking a memory test on older adults' subjective age. Psychological Science, 24(12), 2481-2488.

15. Kogan, N. (1961). Attitudes toward old people: The development of a scale and an examination of correlates. Journal of Abnormal and Social Psychology, 62(1), 44-54.

16. Kornadt, A., Voss, P. \& Rothermund, K. (2015). Age stereotypes and self-views revisited: Patterns of internalization and projection processes across the life span. The Journals of Gerontology Series B: Psychological Sciences and Social Sciences. doi: 10.1093/geronb/gbv099

17. Kotter-Grühn, D. \& Hess, T. (2012). The impact of age stereotypes on self-perceptions of aging across the adult lifespan. The Journals of Gerontology, Series B: Psychological Sciences and Social Sciences, 67(5), 563-571. 
18. Kotter-Grühn, D., Kornadt, A. E., \& Stephan, Y. (2016). Looking beyond chronological age: Current knowledge and future directions in the study of subjective age. Gerontology, 62(1), 86-93.

19. Kotter-Grühn, D., Neupert, S. D., \& Stephan, Y. (2015). Feeling old today? Daily health, stressors, and affect explain day-to-day variability in subjective age. Psychology \& health, 30(12), 1470-1485.

20. Levy, B. (2003). Mind matters: cognitive and physical effects of aging self-stereotypes. Journal of Gerontology: Psychological Sciences, 58B(4), 203-211.

21. Levy, B. (2009). Stereotype embodiment a psychosocial approach to aging. Current Directions in Psychological Science, 18(6), 332-336.

22. Levy, B. R., Ferrucci, L., Zonderman, A. B., Slade, M. D., Troncoso, J., \& Resnick, S. M. (2016). A culture-brain link: Negative age stereotypes predict Alzheimer's disease biomarkers. Psychology and aging, 31(1), 82.

23. Levy, B., Slade, M., Murphy, T. \& Gill, T. (2012). Association between positive age stereotypes and recovery from disability in older persons. The Journal of the American Medical Association, 308(19), $1972-1973$.

24. Levy, B., Zonderman, A., Slade, M. \& Ferrucci, L. (2009). Age stereotypes held earlier in life predict cardiovascular events in later life. Psychological Science, 20(3), 296-298.

25. Robertson, D. A., King-Kallimanis, B. L., \& Kenny, R. A. (2016). Negative perceptions of aging predict longitudinal decline in cognitive function. Psychology and Aging, 31(1), 71.

26. Rothermund, K. \& Brandtstädter, J. (2003). Age stereotypes and selfviews in later life: Evaluating rival assumptions. International Journal of Behavioral Development, 27(6), 549-554.

27. Rupp, D., Vodanovich, S. \& Credé, M. (2005). The multidimensional nature of ageism: construct validity and group differences. Social Psychology, 145(3), 335-362.

28. Sargent-Cox, K., Anstey, K. \& Luszcz, M. (2012). The relationship between change in self-perceptions of aging and physical functioning in older adults. Psychology and aging, 27(3), 750-760.

29. Sargent-Cox, K., Anstey, K. \& Luszcz, M. (2013). Longitudinal change of self-perceptions of aging and mortality. Journals of Gerontology, Series B: Psychological Sciences and Social Sciences, 69(2), 168-173.

30. Sarkisian, C., Prohaska, T., Wong, M., Hirsch, S. \& Mangione, C. (2005). The relationship between expectations for aging and physical activity among older adults. Journal of General Internal Medicine, 20(10), 911-915. 
31. Serrani, D. (2011). El trabajo de observación del adulto mayor. Una herramienta pedagógica para modificar actitudes ageístas en estudiantes de psicología. Revista Electrónica de Investigación Educativa, 13(1), 71-85.

32. Stephan, Y., Chalabaev, A., Kotter-Grühn, D. \& Jaconelli, A. (2012). "Feeling younger, being stronger": an experimental study of subjective age and physical functioning among older adults. The Journals of Gerontology, Series B: Psychological Sciences and Social Sciences, 68(1), 1-7.

33. Stephan, Y., Sutin, A. R., \& Terracciano, A. (2015). How old do you feel? The role of age discrimination and biological aging in subjective age. PloS one, 10(3), e0119293. 